\title{
The Subgraph Similarity Problem
}

\author{
Lorenzo De Nardo, Francesco Ranzato, Francesco Tapparo \\ Dipartimento di Matematica Pura ed Applicata, University of Padova, Italy
}

\begin{abstract}
Similarity is a well known weakening of bisimilarity where one system is required to simulate the other and vice versa. It has been shown that the subgraph bisimilarity problem, a variation of the subgraph isomorphism problem where isomorphism is weakened to bisimilarity, is NP-complete. We show that the subgraph similarity problem and some related variations thereof still remain NP-complete.
\end{abstract}

Index Terms-Graph simulation, subgraph similarity problem, NP-completeness.

\section{INTRODUCTION}

Bisimilarity, weak bisimilarity and similarity are well known behavioural equivalences that arise in every process calculus like CCS, $\pi$-calculus, ambient calculus, etc. [5]. These equivalences are also used as structural indexes to support efficient evaluation of query processing in graph-structured data, e.g. 1-index and $\mathrm{A}(k)$-index for XML graphs [1], [4], [6], [9]. Dovier and Piazza [2] consider the so-called subgraph bisimilarity problem, a variant of the well-known NPcomplete subgraph isomorphism problem where isomorphism is weakened to bisimilarity: this is the problem of identifying a subgraph $G_{2}^{\prime}$ of a graph $G_{2}$ bisimilar to a given graph $G_{1}$. The motivation for considering such a problem arises from data retrieval in query languages like G-log [8]. Dovier and Piazza prove that this problem remains NP-complete by means of a reduction of the Hamiltonian path problem (HP).

Similarity is a well known weakening of bisimilarity where one system is required to simulate the other and vice versa. We show that the subgraph similarity problem remains NPcomplete and we still use a reduction of HP for proving this. On the other hand, weak bisimulation is a weakening of bisimulation that allows to bisimulate one step of a system by means of any finite number of steps. It turns out that weak bisimilarity is stronger than similarity. Thus, as a consequence, we also obtain that the subgraph weak bisimilarity problem is NP-complete.

\section{BACKGROUND}

Let $R \subseteq X \times X$ and $S \subseteq X \times Y$ be binary relations. Then, $R^{+} \subseteq X \times X$ denotes the transitive closure of $R$ and $S^{-1} \subseteq$ $Y \times X$ denotes the inverse relation $\{(y, x) \mid(x, y) \in S\}$. Given a graph $G, N(G)$ and $E(G)$ denote the sets of nodes and edges of $G$. The $n$-chain directed graph $C_{n}$, with $n \geq 1$, is $C_{n}=\left(\left\{x_{1}, \ldots, x_{n}\right\}, \rightarrow\right)$ where $x_{i} \rightarrow x_{i+1}$ for any $i \in[1, n-1]$.

The notions of (weak) simulation and (weak) bisimulation are given for labeled directed graphs in the context of process calculi or model checking, namely labeled transition systems when labels are attached to edges or Kripke structures when labels are attached to the nodes. The corresponding notions of (weak) simulation and (weak) bisimulation for unlabeled directed graphs are obtained as a particular case when one considers a single label. Let $G_{1}=\left(N_{1}, \rightarrow_{1}\right)$ and $G_{2}=\left(N_{2}, \rightarrow_{2}\right)$ be two directed graphs.

- A simulation of $G_{1}$ by $G_{2}$ is a relation $R \subseteq N_{1} \times N_{2}$ such that: (1) $R$ is total, i.e., for any $n \in N_{1}$ there exists $m \in N_{2}$ such that $(n, m) \in R$; (2) if $(n, m) \in R$ and $n \rightarrow{ }_{1} n^{\prime}$ then there exists $m^{\prime} \in N_{2}$ such that $\left(n^{\prime}, m^{\prime}\right) \in R$ and $m \rightarrow_{2} m^{\prime}$.

- A weak simulation of $G_{1}$ by $G_{2}$ is a relation $R \subseteq N_{1} \times N_{2}$ such that: (1) $R$ is total, i.e., for any $n \in N_{1}$ there exists $m \in N_{2}$ such that $(n, m) \in R$; (2) if $(n, m) \in R$ and $n \rightarrow 1 n^{\prime}$ then there exists $m^{\prime} \in N_{2}$ such that $\left(n^{\prime}, m^{\prime}\right) \in R$ and $m \rightarrow{ }_{2}^{+} m^{\prime}$.

- $G_{1}$ and $G_{2}$ are (weakly) bisimilar when there exists a relation $R \subseteq N_{1} \times N_{2}$, called (weak) bisimulation, such that: (1) $R$ is a (weak) simulation of $G_{1}$ by $G_{2} ;(2) R^{-1}$ is a (weak) simulation of $G_{2}$ by $G_{1}$.

- $G_{1}$ and $G_{2}$ are (weakly) similar when there exist two relations $R \subseteq N_{1} \times N_{2}$ and $S \subseteq N_{2} \times N_{1}$ such that: (1) $R$ is a (weak) simulation of $G_{1}$ by $G_{2}$; (2) $S$ is a (weak) simulation of $G_{2}$ by $G_{1}$.

$G_{1} \leq_{\operatorname{sim}} G_{2}$ denotes the fact that there exists a simulation of $G_{1}$ by $G_{2}$, so that $G_{1}$ and $G_{2}$ are similar when $G_{1} \leq_{\text {sim }}$ $G_{2}$ and $G_{2} \leq_{\operatorname{sim}} G_{1}$. Moreover, we denote, respectively, by $G_{1} \sim_{\text {bis }} G_{2}, G_{1} \sim_{\text {wbis }} G_{2}, G_{1} \sim_{\text {sim }} G_{2}$ and $G_{1} \sim_{\text {wsim }} G_{2}$ the fact that $G_{1}$ and $G_{2}$ are, respectively, bisimilar, weakly bisimilar, similar and weakly similar. Clearly, it turns out that $G_{1} \sim_{\text {bis }} G_{2}$ $\Rightarrow G_{1} \sim_{\mathrm{wbis}} G_{2}$ and $G_{1} \sim_{\mathrm{sim}} G_{2} \Rightarrow G_{1} \sim_{\mathrm{wsim}} G_{2}$.

Lemma II.1. $G_{1} \sim_{\text {wsim }} G_{2} \Rightarrow G_{1} \sim_{\text {sim }} G_{2}$ and $G_{1} \sim_{\text {wbis }} G_{2} \Rightarrow$ $G_{1} \sim_{\operatorname{sim}} G_{2}$.

Proof: Let $R \subseteq N_{1} \times N_{2}$ be a weak simulation of $G_{1}$ by $G_{2}$. We consider the relation $R_{+} \subseteq N_{1} \times N_{2}$ obtained by adding to $R$ the following pairs: for any $u, u^{\prime} \in N_{1}$ and $v, v^{\prime} \in$ $N_{2}$ such that $u \rightarrow_{1} u^{\prime},(u, v) \in R, v \rightarrow_{2}^{+} v^{\prime}$ and $\left(u^{\prime}, v^{\prime}\right) \in R$, if $v \rightarrow_{2}^{+} v^{\prime \prime} \rightarrow_{2}^{+} v^{\prime}$ then $\left(u^{\prime}, v^{\prime \prime}\right)$ is added to $R$. Then, it is clear that $R_{+}$is a simulation of $G_{1}$ by $G_{2}$. Moreover, if $R$ is a weak bisimulation between $G_{1}$ and $G_{2}$, we consider the simulations $R_{+}$and $R_{+}^{-1}$ so that $G_{1}$ is similar to $G_{2}$.

Thus, $G_{1} \sim_{\mathrm{bis}} G_{2} \Rightarrow G_{1} \sim_{\text {wbis }} G_{2} \Rightarrow G_{1} \sim_{\text {sim }} G_{2} \Leftrightarrow$ $G_{1} \sim_{\text {wsim }} G_{2}$. The following example shows that the first two implications are actually strict.

Example II.2. Let us consider the following graphs: 


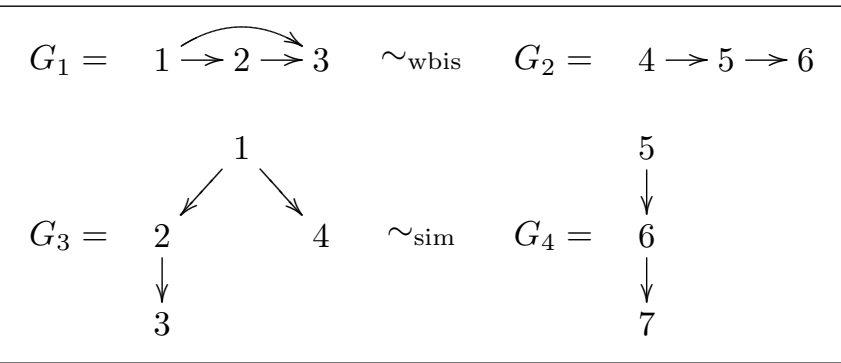

It turns out that $G_{1} \sim{ }_{\text {wbis }} G_{2}$ by the weak bisimulation $R=$ $\{(1,4),(2,5),(3,6)\}$ while there is no bisimulation between $G_{1}$ and $G_{2}$. On the other hand, we have that $G_{3} \sim_{\text {sim }} G_{4}$ by the simulations $R=\{(1,5),(2,6),(3,7),(4,6)\}$ and $S=\{(5,1),(6,2),(7,3)\}$ while there is no weak bisimulation between $G_{3}$ and $G_{4}$.

The subgraph bisimilarity (respectively, weak bisimilarity, similarity) problem, denoted by $\operatorname{Bis}\left(G_{1}, G_{2}\right)$ (respectively, $\left.\operatorname{WBis}\left(G_{1}, G_{2}\right), \operatorname{Sim}\left(G_{1}, G_{2}\right)\right)$, consists of deciding whether there exists a subgraph $G_{2}^{\prime}$ of $G_{2}$ such that $G_{1} \sim_{\text {bis }} G_{2}^{\prime}$ (respectively, $G_{1} \sim_{\text {wbis }} G_{2}^{\prime}, G_{1} \sim_{\operatorname{sim}} G_{2}^{\prime}$ ). The size of an instance of one of such problems is given by $\left|N_{1}\right|+\left|N_{2}\right|+\left|\rightarrow_{1}\right|+\left|\rightarrow_{2}\right|$.

\section{The Subgraph Similarity Problem IS NP-COMPLETE}

Dovier and Piazza [2] show that the subgraph bisimilarity problem Bis is NP-complete by reducing the directed Hamiltonian path problem HP to Bis. The proof is direct and basically depends on the fact that if a $n$-chain is bisimilar to a graph $G$ with $n$ nodes then $G$ actually is isomorphic to the $n$-chain. We also reduce HP to Sim in order to prove that Sim is NP-hard: in this case the proof becomes less direct.

Let us first observe that $\mathrm{Sim}$ is in NP because $G_{2}^{\prime} \sim_{\operatorname{sim}} G_{1}$ can be verified in polynomial time by using one polynomialtime simulation equivalence algorithm like that by Henzinger, Henzinger and Kopke [3] that runs in $O\left(\left(\rightarrow_{1}|+| \rightarrow_{2} \mid\right)\left(\left|N_{1}\right|+\right.\right.$ $\left.\left.\left|N_{2}\right|\right)\right)$. In fact, it is easy to show that similarity of two graphs $G_{1}$ and $G_{2}$ can be verified by a simulation equivalence algorithm on the disjoint union graph $G_{1} \cup G_{2}=\left(N_{1} \cup\right.$ $N_{2}, \rightarrow_{1} \cup \rightarrow_{2}$ ).

Let us now show how HP can be reduced to Sim.

Lemma III.1. If $G \sim_{\operatorname{sim}} C_{n}$ then $C_{n}$ is isomorphic to a subgraph of $G$.

Proof: Let us first show that $G$ is an acyclic graph. Assume, by contradiction, that $a_{1} \rightarrow a_{2} \rightarrow \ldots a_{k} \rightarrow a_{1}$ is a cycle in $G$. Let $R$ be the simulation relation of $G$ by $C_{n}$. Then, there exists $x_{j} \in N\left(C_{n}\right)$ such that $\left(a_{1}, x_{j}\right) \in R$. Since $a_{1} \rightarrow a_{2}$ and $x_{j+1}$ is the unique successor of $x_{j}$, by simulation, we have that $\left(a_{2}, x_{j+1}\right) \in R$. Proceeding in this way, since $\left\{a_{1}, \ldots, a_{k}\right\}$ is a cycle, we would have that $\left(a_{l}, x_{n}\right) \in R$, for some $l \in[1, k]$. Since $a_{l} \rightarrow a_{l+1}$, we therefore would obtain that $x_{n}$ must have a successor, which is a contradiction.

On the other hand, in a similar way, since $C_{n} \leq_{\operatorname{sim}} G$, it must be the case that $G$ contains a path of length $n$. Since $G$ is acyclic, the nodes in this path must be distinct, so that this path is indeed a $n$-chain.
Assume that $G$ has $n$ nodes. If $G$ is similar to $C_{n}$ then $G$ contains a $n$-chain as subgraph but $G$ is not necessarily isomorphic to $C_{n}$. By contrast, if $G$ is bisimilar to $C_{n}$ then $G$ is isomorphic to $C_{n}$ [2].

Theorem III.2. Sim is NP-hard.

Proof: Let us show that the Hamiltonian path problem HP can be reduced to Sim. Let $G$ be a graph with $|N(G)|=n$. It turns out that the problem $\operatorname{HP}(G)$ is equivalent to $\operatorname{Sim}\left(C_{n}, G\right)$. On the one hand, if $G$ admits an Hamiltonian path then such path is isomorphic to the $n$-chain $C_{n}$, so that $G$ contains a subgraph which is similar to $C_{n}$. On the other hand, if $G$ contains a subgraph $G^{\prime}$ which is similar to $C_{n}$ then, by Lemma III.1. $G^{\prime}$ has a subgraph which is a $n$-chain and, since $|N(G)|=n$, this $n$-chain turns out to be an Hamiltonian path in $G$. To conclude we observe that this reduction can be done in polynomial time.

\section{Corollary III.3. WBis is NP-complete.}

Proof: Let $G$ be a graph with $|N(G)|=n$. By the proof of Theorem III.2, $\operatorname{HP}(G) \Leftrightarrow \operatorname{Sim}\left(C_{n}, G\right)$. By the proof of [2, Theorem 1], $\operatorname{HP}(G) \Leftrightarrow \operatorname{Bis}\left(C_{n}, G\right)$. Moreover, $\operatorname{Bis}\left(C_{n}, G\right) \Rightarrow \operatorname{WBis}\left(C_{n}, G\right)$ trivially holds. On the other hand, by Lemma II.1. $\operatorname{WBis}\left(C_{n}, G\right) \Rightarrow \operatorname{Sim}\left(C_{n}, G\right)$. Thus, the Hamiltonian path problem HP can be reduced to WBis.

Let us finally observe that WBis is in NP because $G_{2}^{\prime} \sim_{\text {wbis }} G_{1}$ can be verified in polynomial time by first computing in polynomial time the transitive closure of $G_{2}^{\prime}$ and $G_{1}$ by Warshall's algorithm and then using a polynomial-time bisimulation algorithm like that by Paige and Tarjan [7] that runs in $O\left(\left(\left|\rightarrow_{1}\right|+\left|\rightarrow_{2}\right|\right) \log \left(\left|N_{1}\right|+\left|N_{2}\right|\right)\right)$.

Finally, it is worth remarking that Bis, WBis, Sim and WSim remain NP-complete problems also when considering the notions of (weak) bisimulation/simulation for labeled graphs, as the corresponding unlabeled problems can be reduced to them simply by considering a single label.

\section{REFERENCES}

[1] P. Buneman, S. Davidson, M. Fernandez and D. Suciu. Adding structure to unstructured data. In Proc. $6^{\text {th }}$ ICDT, LNCS 1186, pp. 336-350, 1997.

[2] A. Dovier and C. Piazza. The subgraph bisimulation problem. IEEE Trans. Knowl. Data Eng., 15(4):1055-1056, 2003.

[3] M.R. Henzinger, T.A. Henzinger and P.W. Kopke. Computing simulations on finite and infinite graphs. In Proc. 36 ${ }^{\text {th }}$ FOCS, pp. 453-462, 1995.

[4] R. Kaushik, P. Shenoy, P. Bohannon and E. Gudes. Exploiting local similarity for indexing paths in graph-structured data. In Proc. $18^{\text {th }}$ ICDE, pp. 129-140, 2002.

[5] R. Milner. Communicating and Mobile Systems: the Pi-Calculus. Cambridge University Press, 1999.

[6] T. Milo and D. Suciu. Index structures for path expressions. In Proc. $7^{\text {th }}$ ICDT, LNCS 1540, pp. 277-295, 1999.

[7] R. Paige and R.E. Tarjan. Three partition refinement algorithms. SIAM J. Comput., 16(6):973-989, 1987

[8] J. Paredaens, P. Peelman and L. Tanca. G-Log: A declarative graphical query language. IEEE Trans. Knowl. Data Eng., 7(3):436-453, 1995.

[9] P. Ramanan. Covering indexes for XML queries: bisimulation - simulation = negation. In Proc. $29^{\text {th }}$ VLDB, pp. 165-176, 2003. 